\title{
BMJ Open Relationship between management and resilience in healthcare: a study protocol for a systematic review
}

\author{
Erika E Petersen, ${ }^{1}$ Hilda Bø Lyng (D) , ${ }^{2}$ Eline Ree, ${ }^{2}$ Siri Wiig (i) ${ }^{3}$
}

To cite: Petersen EE, Lyng HB, Ree $\mathrm{E}$, et al. Relationship between management and resilience in healthcare: a study protocol for a systematic review. BMJ Open 2021;11:e047855. doi:10.1136/ bmjopen-2020-047855

- Prepublication history and additional online supplemental material for this paper are available online. To view these files, please visit the journal online (http://dx.doi.org/10. 1136/bmjopen-2020-047855).

Received 13 December 2020 Accepted 28 June 2021

Check for updates

(c) Author(s) (or their employer(s)) 2021. Re-use permitted under CC BY-NC. No commercial re-use. See rights and permissions. Published by BMJ.

${ }^{1}$ Faculty of Health Sciences, Queen's University Faculty of Health Sciences, Kingston, Alberta, Canada

${ }^{2}$ SHARE Centre for Resilience in Healthcare, Department of Quality and Health Technology, University of Stavanger,

Stavanger, Norway

${ }^{3}$ Centre Director SHARE, Centre for Resilience in Healthcare, Department of Quality and Health Technology, University of Stavanger, Stavanger, Norway

Correspondence to

Dr Hilda Bø Lyng;

hilda.b.lyng@uis.no

\section{ABSTRACT}

Introduction Management has been identified as a critical component of organisational resilience when responding to adverse events and crises, as managers must ensure an effective operational response and provide direction and guidance to teams. While there are many management approaches, strategies and interventions that have been applied and studied in healthcare, the impact of them in relation to resilience in healthcare has not been explored, particularly at the organisational level. Understanding the impact of management approaches, strategies and interventions on resilience has the potential to inform healthcare organisations on how to better use management to prepare and respond to organisational adverse events. The objective of this mixed-methods systematic review is to understand the relationship between management and organisational resilience in healthcare, including management approaches and strategies that promote resilience in healthcare. Methods and analysis A search through MEDLINE, Cumulative Index to Nursing and Allied Health Literature, PubMed and EMBASE will be conducted between 1 August 2021 and 31 December 21. This review will consider empirical quantitative, qualitative and mixed-methods studies published in English from 2010 to the present that meet the inclusion and exclusion criteria. Selected studies will be assessed in detail and extracted data will be reviewed by two independent reviewers. Results of the search will be reported in full in the final systematic review and presented in a Preferred Reporting Items for Systematic Reviews and Meta-Analysis flow diagram. This review will follow a convergent integrated approach to data synthesis and integration.

Ethics and dissemination This systematic literature review includes no collection of primary data; hence ethical approval will not be sought. The outcomes from this review will be disseminated in a peer-reviewed journal, as conference presentation, and as condensed summary for managers in healthcare and policy-makers.

PROSPERO registration number CRD42020223362.

\section{INTRODUCTION}

Over the past decade, resilience in healthcare has emerged and is a growing area of study in the field of health quality and safety science. ${ }^{12}$ In healthcare, safety is often associated with quality, which according to Institute of Medicine (2001) includes six dimensions:

\section{Strengths and limitations of this study}

This is the first systematic review investigating the relationship between management strategies and resilience in healthcare.

- The results from this systematic review will provide an overview of what is currently known about the relationship between management and resilience.

- Understanding the impact of management approaches, strategies and interventions on resilience have the potential to inform healthcare organisations on how to better use management to prepare to mitigate risk and respond to organisational adverse events.

- Resilience is a broad field and studies might be missed by the chosen databases.

- We expect to find limited research evidence which may also limit the ability to develop recommendations on management strategies that promote resilience.

patient safety, clinical effectiveness, patientcentredness, coordination, efficiency and equity. ${ }^{3}$ While varying definitions of resilience in healthcare exist, for the purposes of this systematic review, the definition by Wiig et al, will be used to define resilience in healthcare as 'the capacity to adapt to challenges and changes at different system levels, to maintain high quality care, ${ }^{2}$ as it connects resilience in healthcare to quality care.

Resilience in healthcare applies both resilient engineering and safety-II approaches that enable individuals, teams and organisations to adapt to adverse events, challenges and changes. ${ }^{4}$ For example, when faced with adverse events, organisations characterised by resilient performance have a greater ability to adapt to the unexpected challenges while maintaining quality patient care, in comparison to healthcare organisations without these characteristics. ${ }^{5}$

Management has been identified as a critical component of organisational resilience when responding to adverse events and crises, as managers must ensure an effective 
operational response and provide direction and guidance to teams. ${ }^{67}$ While there are many management approaches, strategies and interventions that have been applied and studied in healthcare, the impact of them on promoting resilience in healthcare has not been explored, particularly at the organisational level. ${ }^{8}$ In recent work, Mintzberg states that management and healthcare often have been studied separately in previous research. ${ }^{9}$ As such, there is an ongoing demand for a more detailed understanding of management roles and involvement to improve safety and to achieve quality healthcare services. In terms of management for patient safety and quality in healthcare, focus has often been directed towards the prevention of adverse events (safety-I perspective). ${ }^{10}$ The introduction of resilience therefore provided a new perspective for understanding quality and safety in healthcare with stronger emphasis on how things go right (safety-II perspective). Accordingly, new studies of management are needed to understand the impact of managers in terms of resilience. ${ }^{8}$

However, some studies have identified a connection between management and resilience in healthcare and have suggested there is value in conducting studies to better understand the relationship. ${ }^{611}{ }^{12}$ Specifically, 'the higher the skills of leadership, the higher the ability to be resilient and to overcome challenges'. ${ }^{6}$ Understanding the impact of management approaches, strategies and interventions on resilience at the microlevel, mesolevel and macrolevel has the potential to inform healthcare organisations on how to better use management to prepare and respond to organisational adverse events, challenges and changes.

Healthcare organisations are known for being difficult to control and manage, mainly because they introduce a conflict between clinical logic and administrative logic. ${ }^{13}$ Healthcare managers includes a range of positions from nursing and physician managers close to the front line, to head of departments at the mesolevel, to top management in for example, nursing homes and hospitals. However, in all these different positions healthcare managers are instrumental for balancing and converging policy and front-line practices, and studies of healthcare management is as such a way of exploring both directions. ${ }^{10}$

A preliminary search of PROSPERO, MEDLINE and the Cochrane Database of Systematic Reviews was conducted and, to our knowledge, no current or underway systematic reviews on this topic were identified. While there are systematic reviews pertaining to resilience in healthcare, none exist that specifically target the relationship between management and resilience in healthcare. ${ }^{414} 15$ Patriarca $e t a l$, in their literature review, explores system safety and resilience engineering in terms of anaesthesiological practices. ${ }^{16}$ Iflaifel et al have also provided a valuable review of the resilience literature. Even though management is highlighted as key for enhancing resilience in healthcare in this review, management strategies and approaches are not of focus. ${ }^{4}$ Furthermore, Berg et al described methodological strategies in resilience studies, which differs from our aim of understanding leadership strategies for resilience in this review. ${ }^{14}$ Valuable understanding of the resilience field is also provided by Ellis et al, yet not much attention of management strategies is included. ${ }^{15}$ As such, new systematic literature reviews are needed to provide understanding of the relationship between management and resilience in healthcare. ${ }^{8}$

This systematic review contributes to this by investigating the following objectives:

1. Identify and synthesise studies pertaining to management and resilience in healthcare.

2. Explore the relationship between and impact of management approaches and strategies on promoting resilience in healthcare.

\section{Review questions}

1. What is the relationship between management and resilience in healthcare?

2. What type of management strategies and approaches promote resilience in healthcare?

\section{Inclusion criteria}

This review will consider quantitative, qualitative and mixed-methods studies that evaluate the relationship between management and resilience in healthcare and/ or explore management/leadership activities, strategies, approaches and interventions that promote resilience in healthcare. Specifically, studies considered for inclusion need to be focused on management and resilience. This includes studies with management focus from different perspectives and levels such as managers themselves, but also the perspective of their employees. All studies to be included will be based on empirical data, including data collection and data from observations, interviews (all types) and surveys. Studies published in English from 2010 to the present will be included, as the resilience in healthcare literature was mainly developed from 2012 and onwards. ${ }^{17}$ Only research on organisational resilience will be included, but the impact of organisational resilience for the different levels (micro, meso and macro) is also to be included in this review. Additionally, studies considered for inclusion will include the following outcome measures: (1) the impact of management on resilience in healthcare and (2) the influence of management and resilience in terms of quality outcomes in healthcare. Other outcomes may be considered based on what is discovered during the review, such as adaptive capacity.

\section{Exclusion criteria}

This review will exclude quantitative, qualitative, and mixed methods studies that:

1. Do not focus on management and resilience in healthcare.

2. Focus on non-health disciplines and/or non-healthcare settings.

3. Include emotional, psychological, individual, community or disaster resilience. 
4. Do not include formal leaders and only focus on leaders without a title and/or champions.

5. Are unpublished and/or are opinion pieces, theoretical reflections and articles without methods sections.

\section{METHODS AND ANALYSIS}

The review will be prepared using the Preferred Reporting Items for Systematic Reviews and Meta-Analyses (PRISMA) guidelines for reporting systematic reviews. ${ }^{18}$

\section{Search strategy}

The search strategy will aim to locate published studies. An initial limited search of MEDLINE, Cumulative Index to Nursing and Allied Health Literature (CINAHL), PubMed and Embase was undertaken to identify articles on the topic. The text words contained in the titles and abstracts of relevant articles, as well as the index terms used to describe the articles were used to develop a fullsearch strategy that was tested through MEDLINE (see online supplemental appendix). The search strategy, including all identified keywords and index terms, will be adapted for each included information source. The reference list of all studies selected for critical appraisal will be screened for additional studies.

\section{Information sources}

The following electronic databases will be searched between 1 August 2021 and 31 December 21: MEDLINE, CINAHL, PubMed and EMBASE.

\section{Study selection}

Following the search, all identified citations will be collated and uploaded into EndNote X9 and duplicates removed. Titles and abstracts will then be screened by two independent reviewers for assessment against the inclusion criteria for the review. Potentially relevant studies will be retrieved in full and assessed in detail against the inclusion criteria by two independent reviewers. Reasons for exclusion of full text studies that do not meet the inclusion criteria will be recorded and reported in the systematic review. Any disagreements that arise between the reviewers at each stage of the study selection process will be resolved through discussion, or with a third reviewer. The results of the search will be reported in full in the final systematic review and presented in a PRISMA flow diagram. $^{18}$

An assessment form (including relevance to research questions, the context, data source, informants, type of study) will be designed for the first stage as a framework for decision making on whether studies will be included or excluded. ${ }^{19}$ If the researchers are ambiguous about whether to include a paper after reading the abstract, an assessment of the full article will be performed.

\section{Assessment of methodological quality}

Quantitative, qualitative and mixed-methods studies selected for retrieval will be assessed by two independent reviewers for methodological validity prior to inclusion in the review using the critical appraisal tools developed by the Joanna Briggs Institute. Specifically, for quantitative studies (and quantitative component of mixed methods studies), the two independent reviewers will use the 'Checklist for Randomised Controlled Trials (RCTs), ${ }^{20}$ and/or 'Checklist for Quasi-Experimental Studies ${ }^{21}$ (nonrandomised experimental studies) depending on the type of quantitative study. Further, for qualitative studies (and qualitative component of mixed methods studies), the two independent reviewers will use the 'Checklist for Qualitative Research'. ${ }^{22}$ Additionally, the included studies will also be assessed and categorised in terms of the methodological approach (eg, meta-analysis, RCT, case-control, cross-sectional, case studies, observation, interview, action research).

Any disagreements that arise between the reviewers will be resolved through discussion, or with a third reviewer. The results of critical appraisal will be reported in narrative form and in a table.

Following critical appraisal, studies that do not meet a certain quality threshold will be excluded. This decision will be based on the exclusion criteria and the Quality Assessment Tool.

\section{Data extraction}

Data will be extracted from the quantitative, qualitative and mixed-methods studies included in the review by two independent reviewers. The data will be extracted and incorporated in a framework that will include specific details about the publication (authors, date of publication, country), research question(s), study design (quantitative, qualitative, mixed methods), population (eg, sample size, age, gender, occupational status, managers, employees), context (hospital, nursing homes, home care services, location of the study), culture, study methods, the phenomena of interest relevant to the review objective, contextual level (team, organisation), theoretical underpinnings, management strategy and approach, main findings (management strategies, management approaches, change in clinical practice, change in organisational practice, cultural change, change in use of technology, learning processes, processes of anticipation, monitoring, responding, innovations, adaptations, adaptive capacity, capacity-demands handling and trade-offs) and other relevant data. Findings, and their illustrations will be extracted and assigned a level of credibility.

\section{Data synthesis and integration}

This review will follow a convergent integrated approach for mixed-methods systematic reviews. ${ }^{23}{ }^{24}$ The included quantitative data will be summarised in order to allow for convergence of findings from different methodological approaches. The convergent integrated approach involves assembling the summarised quantitative data with the qualitative data. Assembled data are categorised and pooled together based on similarity in meaning to produce a set of integrated findings in the form of line of action statements. 


\section{Patient and public involvement}

There is no patient and public involvement in the development of the protocol. The following systematic literature review will be presented for the SHARE (Centre for Resilience in Healthcare) research centre's patient and stakeholder panel (PSI panel) and additionally for associated coresearchers within the research centre.

\section{DISCUSSION}

All organisations are in a constant state of change. In order for organisations to manage the need for continuous changes, both resilience and management are described as important factors for succeeding. ${ }^{25}$ It is well known that both management and resilience are important predictors for quality in healthcare. ${ }^{6}$ However, research has mostly studied resilience and management in isolation. ${ }^{7}$ Correspondingly, there has been raised a call for new studies to include management in resilience studies, and thereby a shift of the empirical focus from the traditional microlevel focus within resilience literature, to include mesolevel and macrolevel. ${ }^{414} 15$

To this end, this systematic literature will provide a foundation for new studies combining management and resilience by establishing an overview of what is currently known about this linkage and furthermore implications for new areas of research.

In the major project, Resilience in Healthcare, ${ }^{26}$ that this review is related to, we continue our innovative work to stronger link resilience to healthcare quality to have a larger impact on conceptualising and approaching resilience in healthcare with a multilevel perspective.

\section{ETHICS AND DISSEMINATION}

This study will not collect any primary data and does not require ethical approval.

The results will be published in an international peerreviewed journal and will be shared with stakeholders and at an international conference.

Contributors EEP drafted the protocol with guidance from HBL, ER and SW. EEP, HBL, ER and SW developed a draft of the initial search strategy and contributed to the refinement of the selection criteria. EEP developed the overarching framework for the methodology and drafted the manuscript. SW conceptualised the study and is in lead of the project group. All project members had the opportunity to contribute, revise the manuscript, and have approved the final version.

Funding The authors have not declared a specific grant for this research from any funding agency in the public, commercial or not-for-profit sectors.

Competing interests None declared.

Patient consent for publication Not required.

Provenance and peer review Not commissioned; externally peer reviewed.

Supplemental material This content has been supplied by the author(s). It has not been vetted by BMJ Publishing Group Limited (BMJ) and may not have been peer-reviewed. Any opinions or recommendations discussed are solely those of the author(s) and are not endorsed by BMJ. BMJ disclaims all liability and responsibility arising from any reliance placed on the content. Where the content includes any translated material, BMJ does not warrant the accuracy and reliability of the translations (including but not limited to local regulations, clinical guidelines, terminology, drug names and drug dosages), and is not responsible for any error and/or omissions arising from translation and adaptation or otherwise.

Open access This is an open access article distributed in accordance with the Creative Commons Attribution Non Commercial (CC BY-NC 4.0) license, which permits others to distribute, remix, adapt, build upon this work non-commercially, and license their derivative works on different terms, provided the original work is properly cited, appropriate credit is given, any changes made indicated, and the use is non-commercial. See: http://creativecommons.org/licenses/by-nc/4.0/.

ORCID iDs

Hilda Bø Lyng http://orcid.org/0000-0001-7107-4224

Siri Wiig http://orcid.org/0000-0003-0186-038X

\section{REFERENCES}

1 Kruk ME, Ling EJ, Bitton A, et al. Building resilient health systems: a proposal for a resilience index. BMJ 2017;357:j2323-8.

2 Wiig S, Aase K, Billett S, et al. Defining the boundaries and operational concepts of resilience in the resilience in healthcare research program. BMC Health Serv Res 2020;20:330.

3 Institute of Medicine. Crossing the quality chasm. A New Health System for the $21^{\text {st }}$ Century. Washington: National Academy Press, 2001.

4 Iflaifel M, Lim RH, Ryan K, et al. Resilient health care: a systematic review of conceptualisations, study methods and factors that develop resilience. BMC Health Serv Res 2020;20:1-21.

5 Nemeth C, Wears R, Woods D. Minding the gaps: creating resilience in health care. In: Advances in patient safety: new directions and alternative approaches. 3, 2008. https://www.ncbi.nlm.nih.gov/ books/NBK43670/

6 Tau B, Du Plessis E, Koen D, et al. The relationship between resilience and empowering leader behaviour of nurse managers in the mining healthcare sector. Curationis 2018;41:1-10.

7 Teo WL, Lee M, Lim WS. The relational activation of resilience model: how leadership activates resilience in an organizational crisis. Journal of Contingencies and Crisis Management 2017;25:136-47.

8 Ree E, Ellis LA, Wiig S. Managers' role in supporting resilience in healthcare: a proposed model of how managers contribute to a healthcare system's overall resilience. Int J Health Govern 2021.

9 Mintzberg H. Managing the myths of health care: bridging the separations between care, cure, control, and community. 2017. Berrett-Koehler Publishers, 2017.

10 Ford EW, Savage GT. Patient safety: state-of-the-art in health care management and future directions. Emerald Group Publishing Limited: In Patient Safety and Health Care Management, 2008.

11 Harland L, Harrison W, Jones JR, et al. Leadership behaviors and subordinate resilience. J Leadersh Organ Stud 2005;11:2-14.

12 Nguyen Q, Kuntz JRC, Naswall K. Employee resilience and leadership styles: the moderating role of proactive personality and optimism. New Zealand J Psycho 2016;45:13-21.

13 Andersson T, Gadolin C. The call for leadership in healthcare-what is it we are calling for. Paper presented at the Göteborg Public Management Seminar; November 19-20, Gothenburg, 2013:2013.

14 Berg SH, Akerjordet K, Ekstedt M, et al. Methodological strategies in resilient health care studies: an integrative review. Saf Sci 2018;110:300-12.

15 Ellis LA, Churruca K, Clay-Williams R, et al. Patterns of resilience: a scoping review and bibliometric analysis of resilient health care. Saf Sci 2019;118:241-57.

16 Patriarca R, Gravio GD, Costantino F, et al. A paradigm shift to enhance patient safety in healthcare, a resilience engineering approach: Scoping review of available evidence. Int J Healthcare Technol Manage 2017;16:319-43.

17 Hollnagel Braithwaite \& Wears. ed. Resilient health care. Ashgate. Farnham, 2013.

18 Moher D, Liberati A, Tetzlaff J, et al. Preferred reporting items for systematic reviews and meta-analyses: the PRISMA statement. PLOS Med 2009;6:e1000097.

19 Hawker S, Payne S, Kerr C, et al. Appraising the evidence: reviewing disparate data systematically. Qual Health Res 2002;12:1284-99.

20 The Joanna Briggs Institute. The Joanna Briggs Institute critical appraisal tools for use in JBI systematic reviews: checklist for randomized controlled trials, 2017. Available: https://jbi.global/sites/ default/files/2019-05/JBI_RCTs_Appraisal_tool2017_0.pdf

21 The Joanna Briggs Institute. The Joanna Briggs Institute critical appraisal tools for use in JBI systematic reviews: checklist for quasiexperimental studies (non-randomized experimental studies), 2017. 
Available: https://jbi.global/sites/default/files/2019-05/JBI_QuasiExperimental_Appraisal_Tool2017_0.pdf

22 The Joanna Briggs Institute. The Joanna Briggs Institute critical appraisal tools for use in JBI systematic reviews: checklist for qualitative research, 2017. Available: https://jbi.global/sites/default/ files/2019-05/JBI Critical_Appraisal-Checklist_for_Qualitative_ Research2017_0.pdf

23 Whittemore R, Knafl K. The integrative review: updated methodology. J Adv Nurs 2005;52:546-53.
24 Joanna Briggs Institute. 8.4.1 MMSR questions that take a convergent integrated approach to synthesis and integration, 2020. Available: https://wiki.jbi.global/display/MANUAL/8.4.1+++ MMSR+questions+that+take+a+CONVERGENT+INTEGRATED+approach+to+synthesis+and+integration

25 Allison E. The resilient leader. Educational Leadership 2012;69:79-82.

26 Aase K, Guise V, Billett S, et al. Resilience in healthcare (RiH): a longitudinal research programme protocol. BMJ Open 2020;10:e038779. 
